\title{
Suizidhilfe aus ärztlicher Sicht - die vernachlässigte Fürsorge
}

\section{Gian Domenico Borasio}

Prof. Dr. med., Abteilung Palliativmedizin, Centre Hospitalier Universitaire Vaudois, Lausanne

Für Menschen in verzweifelten Situationen soll es möglich sein, konkrete Hilfsangebote zu erhalten. Versuche, die Suizidhilfe gesetzlich zu regeln, sind bisher gescheitert. Der Gesetzgeber sollte dafür Sorge tragen, dass in dieser hochsensiblen Thematik nicht weiter einseitig der Autonomiebereich - derzeit de facto delegiert an Suizidhilfe-Organisationen - gestärkt wird, sondern auch der gleichwertige Pol der Fürsorge die überfällige legislative Anerkennung erfährt.

In ganz Europa findet derzeit eine intensive Debatte über das Lebensende statt. In England, Frankreich, Deutschland und Österreich diskutieren Politiker sowie Vertreter verschiedener Weltanschauungen und Professionen intensiv über mögliche Änderungen der im jeweiligen Land bestehenden Rechtspraxis. In diesem Zusammenhang ist die mögliche Rolle der Ärzteschaft bei der Vorbereitung, Durchführung und/oder Begleitung von Massnahmen der Sterbehilfe (Tötung auf Verlangen oder Suizidhilfe) besonders umstritten.

Die Aufgabe, in solch verzweifelten Situationen ein konkretes Hilfsangebot zu machen, wurde nicht wahrgenommen.

In diesem Artikel soll zunächst eine kurze Übersicht der vorhandenen empirischen Daten zu Tötung auf Verlangen und assistiertem Suizid in den wichtigsten Ländern und Regionen, die diese Praktiken bisher erlauben, dargestellt werden. Darauf basierend, werden einige Gedanken zur möglichen Entwicklung in der Schweiz und Argumente für eine nationale Gesetzgebung zur Diskussion gestellt.

\section{Empirische Daten}

Wenn man die Zahlen aus denjenigen Staaten anschaut, welche die Tötung auf Verlangen und/oder den assistierten Suizid erlauben, ergibt sich ein interessantes Bild (Abb. 1). In Ländern, die beide Praktiken erlauben, wird die Tötung auf Verlangen ungleich häufiger durchgeführt. In den Niederlanden starben im Jahr 201228 von 1000 Menschen durch Tötung auf Verlangen, aber nur einer von 1000 durch Suizidhilfe. In Bel- gien liegt der Anteil von Todesfällen durch Suizidhilfe nach den vorhandenen Schätzungen noch niedriger. Obwohl die Häufigkeit der Tötung auf Verlangen in den Niederlanden und Belgien in den letzten Jahren deutlich gestiegen ist, bleibt der assistierte Suizid in diesen Ländern eine Seltenheit.

Das klare Überwiegen der Tötung auf Verlangen im Vergleich zur Suizidhilfe dort, wo beides erlaubt ist, deutet an, dass Patienten es vorziehen, wenn Ärzte ihre Tötung vornehmen. Dass die Häufigkeit lebensverkürzender Massnahmen in Belgien und den Niederlanden viel höher ist im Vergleich zu Regionen, in denen nur die Suizidhilfe erlaubt ist, weist zudem darauf hin, dass die Verfügbarkeit von ärztlich durchgeführter Tötung auf Verlangen die psychologische Hemmschwelle für die Bitte um Lebensverkürzung ganz erheblich senkt. Auch vor diesem Hintergrund erscheint die Strafbarkeit der Tötung auf Verlangen als weiterhin gerechtfertigt und geboten.

Die Schweiz, die nur die Suizidhilfe toleriert, sie aber nicht gesetzlich geregelt hat, zeigt eine im Ansatz ähnliche «Wachstumskurve» wie Belgien und die Niederlande, wenn auch auf niedrigerem Niveau. Eine Erklärung hierfür könnte das Fehlen prozeduraler Regelungen und Sorgfaltsbedingungen sein, zumal in den letzten Jahren eine zunehmende Anzahl von Menschen ohne körperliche Erkrankung Suizidhilfe in der Schweiz in Anspruch genommen hat. In Oregon, wo ärztliche Suizidhilfe nur für Schwerstkranke unter streng geregelten Bedingungen möglich ist, zeigt sich hingegen über die Jahre nur ein geringfügiger Anstieg der Fälle von Suizidhilfe mit einer Häufigkeit von weniger als einem Zehntel im Vergleich zur Tötung auf Verlangen in den Niederlanden. 


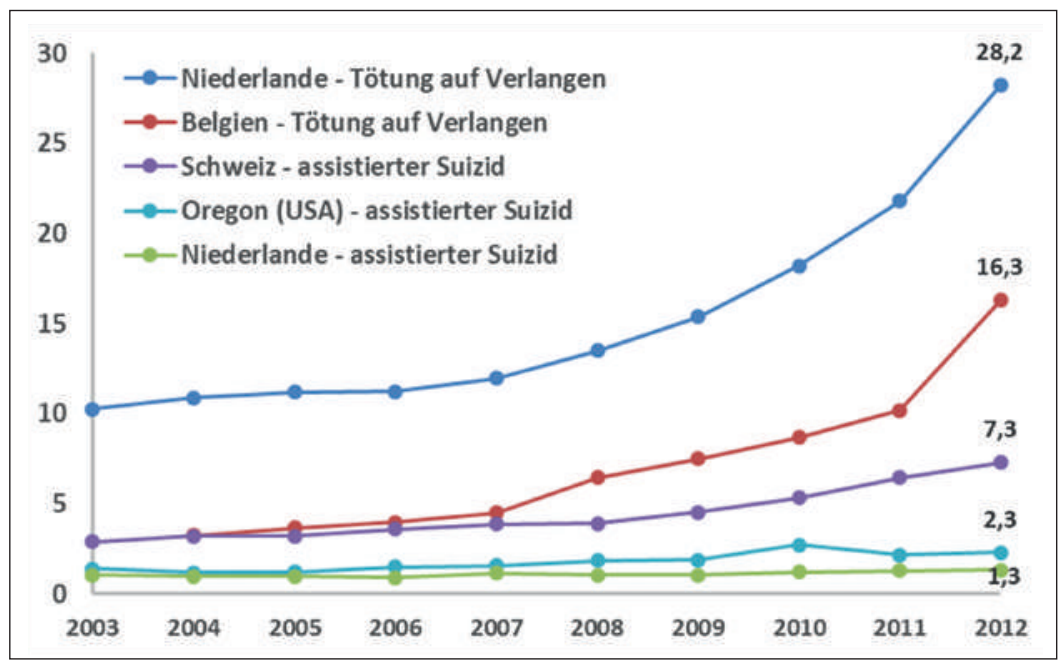

Abbildung 1: Jährliche Häufigkeit (pro 1000 Todesfälle) von Tötung auf Verlangen und assistiertem Suizid in ausgewählten Staaten (Abb. aus Referenz 4).

\section{Gedanken zur Entwicklung in der Schweiz}

Der Verfasser arbeitet erst seit vier Jahren in der Schweiz und kann nicht für sich beanspruchen, die Details der bisherigen Entwicklung ausreichend zu überblicken. Aus dem Vergleich mit anderen Ländern erscheint es jedoch auch für die Schweiz zumindest als denkbar, dass die Ausweitung der Suizidhilfe auf Hochbetagte und chronisch Kranke, wie sie neuerdings von «Exit» propagiert wird [1], negative Folgen zeitigt. Aufgrund immanenter ökonomischer Mechanismen könnte dies schrittweise zu einer Situation führen, in der alte und pflegebedürftige Menschen sich dafür rechtfertigen müssten, wenn sie die Option der Suizidhilfe nicht wahrnehmen und damit die Gemeinschaft «belasten».

Vor einer solchen Entwicklung kann aus ärztlicher Sicht nur gewarnt werden. Hochbetagte und chronisch Kranke haben aufgrund ihrer Situation oft mit Einschränkungen ihrer Autonomie zu kämpfen. Ihre Motorik ist in der Regel reduziert, sie leiden zunehmend unter sensorischen und kognitiven Defiziten (selbst wenn aus juristischer Sicht ihre Urteilsfähigkeit noch erhalten ist) und werden nicht umsonst mit dem Adjektiv «fragil», zu Deutsch zerbrechlich (weil gebrechlich), beschrieben. Diese Menschen, die wir einmal alle selbst sein werden, sind ganz besonders auf die Hilfe und Fürsorge ihrer Familien und der Gesellschaft als Ganzes angewiesen.

\section{Selbstbestimmung und Fürsorge: der fehlende Ausgleich}

Autonomie und Fürsorge stehen bisweilen in einem Spannungsverhältnis zueinander. Jeder Patient braucht vom Arzt seine ganz persönliche «Mischung» aus Fürsorge und Respekt für seine Selbstbestimmung. Das «Mischungsverhältnis» kann sich im Verlauf einer schweren Krankheit durchaus verändern - meist eher in Richtung Fürsorge. Die Patienten immer genau dort abzuholen, wo sie stehen, ist einer der zentralen Aspekte der ärztlichen Kunst. Wie der dänische Philosoph Søren Kierkegaard sagt: «Wenn wir jemandem helfen wollen, müssen wir zunächst herausfinden, wo er steht. Das ist das Geheimnis der Fürsorge. Wenn wir das nicht tun können, ist es eine Illusion zu denken, wir könnten anderen Menschen helfen. Jemandem zu helfen impliziert, dass ich mehr verstehe als er, aber ich muss zunächst verstehen, was er versteht» [2].

In der Schweiz hat der Staat, in Bezug auf die Frage der Suizidhilfe, den Aspekt der Selbstbestimmung de facto (mangels eigener gesetzgeberischer Tätigkeit) den Suizidhilfe-Organisationen überlassen. Diese arbeiten meist sehr gewissenhaft innerhalb der Grenzen, die ihnen das Schweizer Strafrecht (Art. 115 SGB) und die jeweilige kantonale Staatsanwaltschaft ziehen. Da diese Organisationen das Prinzip der Selbstbestimmung verkörpern, erstaunt es nicht, dass sie die Tendenz zeigen, diese «Grenzen» nach Möglichkeit immer mehr

Die Patienten dort abzuholen, wo sie stehen, ist ein zentraler Aspekt der ärztlichen Kunst.

auszuweiten, zuletzt mit dem Vorstoss in Richtung Suizidhilfe für Hochbetagte. Dagegen ist auch grundsätzlich nichts zu sagen, spiegelt die Aktivität der Suizidhilfe-Organisationen doch eine hohe gesellschaftliche Akzeptanz ihrer Praktiken und ein starkes Bedürfnis nach Selbstbestimmung wider. Doch was ist mit dem Gegenpol, der Fürsorge?

Hier hat aus meiner Sicht die schweizerische Gesetzgebung bisher versagt. Alle Versuche einer gesetzlichen Regelung der Suizidhilfe sind in den letzten Jahren gescheitert. Damit wurde die Aufgabe, den Menschen in solch verzweifelten Situationen ein konkretes Hilfsangebot zu machen, nicht wahrgenommen. Fürsorge ist aber eine zentrale Pflicht des Staates. Eine der wichtigsten Aussagen der eidgenössischen Bundesverfassung ist, "dass die Stärke des Volkes sich misst am Wohl der Schwachen». Zwar wurden in den letzten Jahren in wegweisenden und zum Teil erfolgreichen föderalen Programmen die Suizidprävention und die Palliative Care gestärkt. Aber die Menschen, die so sehr unter ihrer Lebenssituation leiden, dass sie um Suizidhilfe bitten, betrachten in der Regel die Suizidhilfeorganisationen als ihre primären Ansprechpartner. Und diese können den Aspekt der Fürsorge nicht abde- 
cken, denn dazu haben sie weder das Mandat noch die Kompetenz.

\section{Überlegungen zu einem Gesetzes- vorschlag}

Es gibt eine Alternative zur legislativen Lethargie in Sachen Suizidhilfe. Sie hat die Waadt vorgemacht. Das neue kantonale Gesetz, das nach Volksentscheid am 1.1.2013 in Kraft getreten ist, regelt die Durchführung der Suizidhilfe in Krankenhäusern und Altenheimen. Dabei werden mehrere Sorgfaltspflichten aufgeführt: Unter anderem muss der behandelnde Arzt informiert werden, und es müssen Hilfestellungen, insbesondere die Palliativmedizin, angeboten werden.

Das Gesetz wurde, trotz seiner unbestrittenen Komplexität, mit grosser Mehrheit angenommen - und zwar anstelle des ursprünglichen Vorschlags von Exit A.D.M.D. (Association pour le Droit de Mourir dans la

\section{Ein Gesetz, das vor der Suizidhilfe eine Beratung durch qualifizierte Ärzte vorsieht, wäre ein grosser Schritt nach vorne.}

Dignité), der sehr einfach formuliert war und nur darauf zielte, Altenheimbewohnern den uneingeschränkten Zugang zur Suizidhilfe zu ermöglichen. Dieses Abstimmungsergebnis beweist, dass die Bevölkerung sehr wohl unterscheiden kann zwischen paternalistischen Gesetzesinitiativen (die darauf abzielen, die Entscheidungsfreiheit der Menschen einzuschränken) und solchen, die vom Geist der Fürsorge getragen sind. Fürsorge heisst nicht, den Menschen ihre Entscheidung abzunehmen, sondern sie in die Lage zu versetzen, informierte Entscheidungen zu treffen. Insofern wäre ein Bundesgesetz, das vor Durchführung einer Suizidhilfe eine Beratung durch qualifizierte Ärzte vorsieht (je nach Situation zum Beispiel Geriater, Psychogeriater, Palliativmediziner oder auch speziell ausgebildete Psychotherapeuten), ein grosser Schritt nach vorne in den Bemühungen, das bislang vernachlässigte Fürsorgeprinzip wieder zur Geltung kommen zu lassen.

\section{Die Rolle der Ärzteschaft}

Die Schweizer Ärzteschaft, vertreten durch ihre wichtigsten Gremien, die FMH und die SAMW, hat sich über die Jahre wiederholt und mit grosser Sorgfalt der Thematik der Suizidhilfe angenommen. In einer neuen nicht repräsentativen - Umfrage aus 2014 zeigt sich eine ambivalente Haltung: für $78 \%$ der antwortenden Ärztinnen und Ärzte ist Suizidhilfe am Lebensende grundsätzlich vertretbar, aber weniger als die Hälfte wäre bereit, Suizidhilfe selbst durchzuführen [3]. Nun steht eine Revision der SAMW-Richtlinie an. Eine der zentralen Fragen lautet, ob der Grundsatz, nach dem die Suizidhilfe keine ärztliche Aufgabe sei, aufrechterhalten bleiben soll. Die Diskussion dürfte spannend werden. Unabhängig von deren Ausgang ist aus meiner persönlichen Sicht als Arzt und Palliativmediziner unstrittig, dass in jedem Fall die Beratung über mögliche lebensorientierte Alternativen zum Suizid eine ur-ärztliche Aufgabe ist - getreu der alten Beschreibung des Arztberufs: "Heilen manchmal, lindern oft, trösten immer». Der Gesetzgeber sollte dafür Sorge tragen, dass in dieser hochsensiblen Thematik nicht weiter einseitig der Autonomiebereich gestärkt wird, sondern auch der gleichwertige Pol der Fürsorge die überfällige legislative Anerkennung erfährt. Die Ärzteschaft kann und sollte in diesem Prozess eine aktive Rolle spielen.

Literatur

1 Exit deutsche Schweiz. GV stimmt pro Altersfreitod. 24.5.2014 www.exit.ch/news/news/details/kommen-sie-zur-gv/ Kommentar dazu: Vögeli D. Alterssuizid erleichtern? Exits Spiel mit dem Feuer. Neue Zürcher Zeitung, 17.5.2014. www.nzz.ch/ zuerich/kommentare/1.18304153

2 Kierkegaard S. Synspunkter for min Forfatter Virksomhet (Der Gesichtspunkt für meine Wirksamkeit als Schriftsteller). In: Die Schriften über sich selbst. Regensburg: Eugen Diederichs Verlag; 1951. S. 38-9.

3 SAMW-Studie Haltung der Ärzteschaft zur Suizidhilfe. Schweiz Ärztezeitung. 2014;95( 47):1767-9.

4 Borasio GD. Selbst bestimmt sterben. München: C.H. Beck; 2014, S. 104. Abbildung modifiziert aus: Gamondi C, Borasio GD, Limoni C, Preston N, Payne S. Legalisation of assisted suicide: a safeguard to euthanasia? Lancet. 2014;384:127. 